\title{
Queuing Models and Subspace Identification in Logistics
}

\author{
T. Vadvári, P. Várlaki \\ Széchenyi István University, System Theory Laboratory \\ Egyetem tér 1., 9026 Győr, Hungary \\ E-mail: varlaki@sze.hu
}

Abstract: In modern logistics it might be helpful to describe the behavior of a complex logistical process as well as to determine the strength of relations between certain parameters of the system. In this paper a subspace identification approach has been applied to estimate the relation between the features of the system based on measured input-output pairs. In order to validate the suitability of the approach for logistical processes a queuing based model has been proposed and used to generate simulation data. Our analysis as well as the obtained results clearly reflect that subspace identification approaches can advantageously be applied to model the relation between certain parameters of the system, nevertheless to characterize the strength of this relation, as well.

Keywords: $\quad$ subspace identification, queuing models, supply chains, modeling

\section{Introduction}

As in many fields also in logistics system modeling and identification approaches play significant role especially when accurate models of complex logistical processes (LP) are needed. Such models may be helpful to predict various features related to the modeled system, such as the response time or in case of supply chains the delivery cycle time, customer order path (related to time spent in different channels), etc. A framework to promote the better understanding of supply chain performance measurement and metrics can be followed for example in [8]. Trough monitoring of performance metrics analytic or statistical models of the observed LP can be designed.

Depending on the knowledge about the modeled system a broad range of solutions can be utilized. Since complex logistical systems are non-linear MIMO systems and are influenced by many parameters their modeling is not a trivial task. Many methods have been proposed to deal with multi-input, multi-output systems in the literature. Perhaps the most popular tool in this topic is the linear parameter varying (LPV) structure by which 
non-linear systems can be modeled and controlled on the basis of linear control theories. If there is no knowledge about the inner structure of the logistic system such as for instance the concrete service strategy and other internal mechanisms only black box like solutions (mainly heuristic approaches) are utilized. In this case the system might be identified based on measured input-output data. In the literature many models (as for instance scheduling, transportation planning, flow-shop sequencing problem) related to logistic systems are based on the fuzzy set and fuzzy control theory [2][3], statistics or their combination [9][10]. Furthermore, the most recent results of the numerical algebra, such as the higher order singular value decomposition and the related tensor product transformation (making connection between LPV models and higher order tensors) offer promising tools to bridge heuristic and analytic approaches. In such a joint framework besides analytic description of the system the expert knowledge can be considered, as well. This may further improve the effectiveness and extend the applicability of the related methods [7][6].

Many times it is difficult to find a proper mathematical model in form of differential equations which would suitable approximate the behavior of the observed logistical process even if the identification of the system is considered locally. However subspace identification techniques combined with tensor product transformation seem to be promising to model complex logistical processes based on input-output data. In this case there is no need for an explicit model parametrization, which is a rather complicated matter for multi-output linear systems [1]. During this research our motivation was first of all to investigate and to show how efficiently subspace identification techniques can be used in case of systems where long delays may occur. We have been focusing first of all on logistical processes which are good examples of such systems. In order to accurately identify the system on subspace basis the input must be persistently exciting, i.e. it must contain sufficiently many distinct frequencies. The Gaussian white noise, pseudo-random binary noise, etc. are the most suitable choices for input. However in case of a logistical process the arrival of demands is considered to be a Poisson process. However arriving demands usually enter the queue of waiting demands which acts like a 'damper' thus to take directly the arrival of demands as input (depending on the size of the mentioned queue and delays in the system) is many times not suitable for identification. Therefore instead of taking the direct input, during the experiments a transformed input has been considered Such a transformed input is efficient even in case when the arrival of demands is modeled by a Poisson or other type of processes. The main contribution of the paper is to show how efficiently a transformed input can be used to identify its relation to certain parameters of the logistical process. In addition it will be shown how the strength of these relations can be characterized with the help of the identified model.

The paper is organized as follows: Section 2 gives a brief overview of subspace identification for deterministic case, Section 3 deals with supply chain models in relation to subspace identification. In Section 4 and 5 examples are reported together with model validation, Section 6 points out the possibilities of embedding logistical processes into linear parameter varying (LPV) framework, finally conclusions and future works are reported. 


\section{Overview on Subspace Identification of LTI Systems}

Before turning the focus onto logistical processes, let us give a brief description on how subspace identification techniques can be used to identify linear time invariant (LTI) vertex models in the parameter space. Let us assume that the local behavior of the logistical system is deterministic, thus it can be described in the well known state space form as follows:

$$
\begin{gathered}
\mathbf{x}_{k+1}=\mathbf{A} \mathbf{x}_{k}+\mathbf{B} \mathbf{u}_{k} \\
\mathbf{y}_{k}=\mathbf{C} \mathbf{x}_{k}+\mathbf{D} \mathbf{u}_{k},
\end{gathered}
$$

where $\mathbf{x}_{k} \in \mathbb{R}^{n}$ stands for the state vector, $\mathbf{u}_{k}$ and $\mathbf{y}_{k}$ represent the input and output vector respectively at time $k$. The goal is to find the model matrices $\mathbf{A}, \mathbf{B}, \mathbf{C}$ and $\mathbf{D}$ based on input-output pairs. As described in [1] let us first arrange the input-output pairs into so called Hankel matrices (reflecting the history of our input-output data):

$$
\begin{gathered}
\mathbf{U}_{1 \mid i}=\left[\begin{array}{cccc}
\mathbf{u}_{1} & \mathbf{u}_{2} & \ldots & \mathbf{u}_{j} \\
\mathbf{u}_{2} & \mathbf{u}_{3} & \ldots & \mathbf{u}_{j-1} \\
\vdots & \vdots & \ldots & \vdots \\
\mathbf{u}_{i} & \mathbf{u}_{i+1} & \ldots & \mathbf{u}_{j+i-1}
\end{array}\right], \\
\mathbf{Y}_{1 \mid i}=\left[\begin{array}{cccc}
\mathbf{y}_{1} & \mathbf{y}_{2} & \ldots & \mathbf{y}_{j} \\
\mathbf{y}_{2} & \mathbf{y}_{3} & \ldots & \mathbf{y}_{j-1} \\
\vdots & \vdots & \ldots & \vdots \\
\mathbf{y}_{i} & \mathbf{y}_{i+1} & \ldots & \mathbf{y}_{j+i-1}
\end{array}\right],
\end{gathered}
$$

and let the history of states (unknown) to be estimated encode as follows:

$$
\mathbf{X}_{i}=\left[\begin{array}{llll}
\mathbf{x}_{i} & \mathbf{x}_{i+1} & \ldots & \mathbf{x}_{i+j-1}
\end{array}\right] .
$$

It can be recognized from (2) that all row vectors in $\mathbf{Y}_{1 \mid i}$ are in the vector space determined by the union of row space of $\mathbf{X}_{i}$ and $\mathbf{U}_{1 \mid i}$. Let us assume that the intersection of row space of $\mathbf{X}_{i}$ and $\mathbf{U}_{1 \mid i}$ is empty. The most simple alternative for estimating $\mathbf{X}_{i}$ (up to a constant multiple $\mathbf{C}$ ) is to project the row space of $\mathbf{Y}_{i}$ onto orthogonal complement of the row space of $\mathbf{U}_{1 \mid i}$. The elements of $\mathbf{Y}_{i}$ can be expressed with the help of the extended observability matrix $\boldsymbol{\Gamma}_{\mathbf{i}}$ and lower block triangular Toeplitz matrix $\mathbf{H}_{i}$ form as follows [1]:

$$
\mathbf{Y}_{1 \mid i}=\boldsymbol{\Gamma}_{\mathbf{i}} \mathbf{X}_{\mathbf{1}}+\mathbf{H}_{\mathbf{i}} \mathbf{U}_{\mathbf{1} \mid \mathbf{i}}
$$

where

$$
\boldsymbol{\Gamma}_{\mathbf{i}}=\left[\begin{array}{llll}
\mathbf{C} & \mathbf{C A} & \ldots & \mathbf{C A}^{i-1}
\end{array}\right]^{\top}
$$


and

$$
\mathbf{H}_{\mathbf{i}}=\left[\begin{array}{ccccc}
\mathbf{D} & 0 & 0 & \ldots & 0 \\
\mathbf{C B} & \mathbf{D} & 0 & \ldots & 0 \\
\mathbf{C A B} & \mathbf{C B} & \mathbf{D} & \ldots & 0 \\
\mathbf{C A}^{i-2} \mathbf{B} & \mathbf{C A}^{i-3} \mathbf{B} & \ldots & \mathbf{C B} & \mathbf{D}
\end{array}\right] .
$$

By substituting recursively into (1) we can express the state sequence $\mathbf{X}_{i+1}$ as follows:

$$
\mathbf{X}_{i+1}=\mathbf{A}^{i} \mathbf{X}_{1}+\boldsymbol{\Delta}_{i} \mathbf{U}_{1 \mid i},
$$

where

$$
\boldsymbol{\Delta}_{i}=\left[\begin{array}{lllll}
\mathbf{A}^{i-1} \mathbf{B} & \mathbf{A}^{i-2} \mathbf{B} & \ldots & \mathbf{A B} & \mathbf{B}
\end{array}\right]
$$

stands for the reversed extended controllability matrix [1]. From (6) the state sequence $\mathbf{X}_{1}$ can be expressed as:

$$
\mathbf{X}_{1}=\Gamma_{i}^{*} \mathbf{Y}_{1 \mid i}-\Gamma_{i}^{*} \mathbf{H}_{i} \mathbf{U}_{1 \mid i},
$$

By substituting (11) into (9) we obtain:

$$
\mathbf{X}_{i+1}=\mathbf{A}^{i} \boldsymbol{\Gamma}_{i}^{*} \mathbf{Y}_{1 \mid i}-\mathbf{A}^{i} \boldsymbol{\Gamma}^{*}{ }_{i} \mathbf{H}_{i} \mathbf{U}_{1 \mid i}+\boldsymbol{\Delta}_{i} \mathbf{U}_{1 \mid i} .
$$

Let us express $\mathbf{X}_{i+1}$ as the sum of two matrices, where one of the matrices contains only the input-output values, i.e.

$$
\mathbf{X}_{i+1}=\mathbf{L}_{i} \mathbf{W}_{1 \mid i}
$$

where

$$
\mathbf{L}_{i}=\left[\boldsymbol{\Delta}_{i}-\mathbf{A}^{i} \boldsymbol{\Gamma}^{*}{ }_{i} \mathbf{H}_{i} \quad \mathbf{A}^{i} \boldsymbol{\Gamma}^{*}{ }_{i}\right]
$$

and

$$
\mathbf{W}_{1 \mid i}=\left[\begin{array}{ll}
\mathbf{U}_{1 \mid i} & \mathbf{Y}_{1 \mid i}
\end{array}\right]^{\top}
$$

Since based on (6)

$$
\mathbf{Y}_{i+1 \mid 2 i}=\boldsymbol{\Gamma}_{\mathbf{i}} \mathbf{X}_{\mathbf{i}+\mathbf{1}}+\mathbf{H}_{\mathbf{i}} \mathbf{U}_{\mathbf{i}+\mathbf{1} \mid \mathbf{2} \mathbf{i}}=\boldsymbol{\Gamma}_{\mathbf{i}} \mathbf{L}_{\mathbf{i}} \mathbf{W}_{\mathbf{1} \mid \mathbf{i}}+\mathbf{H}_{\mathbf{i}} \mathbf{U}_{\mathbf{i}+\mathbf{1} \mid \mathbf{2} \mathbf{i}}
$$

Let us now project $\mathbf{Y}_{i+1 \mid 2 i}$ onto orthogonal complement of $\mathbf{U}_{i+1 \mid 2 i}$. Since the projection of $\mathbf{H}_{i} \mathbf{U}_{i+1 \mid 2 i}$ onto its orthogonal complement is empty subspace we obtain [1]:

$$
\begin{gathered}
\mathbf{Y}_{i+1,2 i} / \mathbf{U}_{i+1,2 i}^{\perp}=\boldsymbol{\Gamma}_{i} \mathbf{L}_{i} \mathbf{W}_{1 \mid i} / \mathbf{U}_{i+1,2 i}^{\perp} \\
\left(\mathbf{Y}_{i+1,2 i} / \mathbf{U}_{i+1,2 i}^{\perp}\right)\left(\mathbf{W}_{1 \mid i} / \mathbf{U}_{i+1,2 i}^{\perp}\right)^{-1}=\boldsymbol{\Gamma}_{i} \mathbf{L}_{i}, \\
\underbrace{\left(\mathbf{Y}_{i+1,2 i} / \mathbf{U}_{i+1,2 i}^{\perp}\right)\left(\mathbf{W}_{1 \mid i} / \mathbf{U}_{i+1,2 i}^{\perp}\right)^{-1} \mathbf{W}_{1 \mid i}}_{\mathbf{O}_{i+1}}=\boldsymbol{\Gamma}_{i} \underbrace{\mathbf{L}_{i} \mathbf{W}_{1 \mid i}}_{\mathbf{X}_{i+1}}, \\
\mathbf{O}_{i+1}=\boldsymbol{\Gamma}_{i} \mathbf{X}_{i+1}
\end{gathered}
$$

Let us investigate the structure of $\mathbf{O}_{i+1}$. Based on (7) and (5) it can be expressed as: 


$$
\mathbf{O}_{i+1}=\left[\begin{array}{llll}
\mathbf{C} & \mathbf{C A} & \ldots & \mathbf{C A}^{i-1}
\end{array}\right]^{\top}\left[\begin{array}{llll}
\mathbf{x}_{i+1} & \mathbf{x}_{i+2} & \ldots & \mathbf{x}_{i+j}
\end{array}\right] .
$$

Based on (21) the rank of $\mathbf{O}_{i+1}$ equals to the rank of the state sequence matrix $\mathbf{X}_{i+1}$. Equivalently, the dimensionality of the state vector $\mathbf{x}$ equals to the dimensionality of $\mathbf{O}_{i+1}$. The rank of $\mathbf{O}_{i+1}$ can be determined by singular value decomposition (SVD) as follows[1]:

$$
\begin{gathered}
\mathbf{O}_{i+1}=\mathbf{U}_{1} \mathbf{S}_{1} \mathbf{V}_{1} \\
\boldsymbol{\Gamma}_{i} \mathbf{X}_{i+1}=\mathbf{U}_{1} \mathbf{S}_{1}^{1 / 2} \mathbf{T T}^{-1} \mathbf{S}_{1}^{1 / 2} \mathbf{V}_{1},
\end{gathered}
$$

where $\mathbf{T}$ is an arbitrary invertible square matrix represen-ting a similarity transformation.

$$
\begin{gathered}
\mathbf{X}_{i+1}=\mathbf{T}^{-1} \mathbf{S}_{1}^{1 / 2} \mathbf{V}_{1} \\
\widetilde{\mathbf{X}}_{i+1}=\mathbf{S}_{1}^{1 / 2} \mathbf{V}_{1}
\end{gathered}
$$

The system matrix can be estimated in the least squares sense from the following set of equations:

$$
\left[\begin{array}{l}
\widetilde{\mathbf{X}}_{i+2} \\
\mathbf{Y}_{i+1}
\end{array}\right]=\left[\begin{array}{ll}
\widetilde{\mathbf{A}} & \widetilde{\mathbf{B}} \\
\widetilde{\mathbf{C}} & \widetilde{\mathbf{D}}
\end{array}\right]\left[\begin{array}{l}
\widetilde{\mathbf{X}}_{i+1} \\
\mathbf{U}_{i+1}
\end{array}\right],
$$

where $\mathbf{U}_{i+1}$ and $\mathbf{Y}_{i+1}$ are input and output block Hankel matrices, respectively having one block row.

\section{Modeling Supply Chains on Subspace Basis}

In this section let us introduce our proposed queuing model suitable to describe and analyze supply chains or loading systems. As depicted in Fig. 1 the system is composed of resource pools, queues, servicing processes. Incoming customers or demands stand for the input of the system. The resource pools are categorized according to the type of the resource. To each resource pool a FIFO queue is connected. Resources waiting in the resource queue are assigned to demands (depending on the service the customer is requesting for). To each service a servicing time is assigned. Let us denote it $T_{S}$. After servicing a given demand the corresponding customer can leave the system while the used resources are released and directed back to the pool of resources of the given type. Servicing a request might be considered as an oriented graph where the nodes represent sub-services and the edges correspond to the ordering and delay of execution between these nodes.

By using such a concept various types of supply chains can be simulated and analyzed. For simplicity in the followings let us assume that services are composed of one node. However the same approach might be applied for services divided to numerous sub-services, 
as well. During this investigation our main goal was to show how queuing models can be identified based on input-output data by using subspace identification techniques.

As already mentioned in the introduction the arrival of demand is modeled by a pseudorandom binary noise however due to the queue of waiting demands (acting like a 'damper') its impact on the system behavior is less significant (depending on the internal structure and parameters of the system). Thus several kind of processes (Poisson process, burst arrival, etc.) can be used to model the arrival of demands, due to long delays it does not significantly affect the efficiency of the identification. Therefore during these experiments a transformed input has been considered (see later in this section). In addition, identification based on input-output data might also be suitable to evaluate the strength of relation between certain parameters of the system.

The parameters of the system are for instance the queue lengths $L_{q i}$, number of resources $N_{R i}$, servicing times $T_{S j}$, where $i$ and $j$ stand for the number of resource pools and number of sub-services, respectively. In the following sections let us investigate a structure where $i=1$ and $j=1$, i.e. there is only one type of resource and one service.

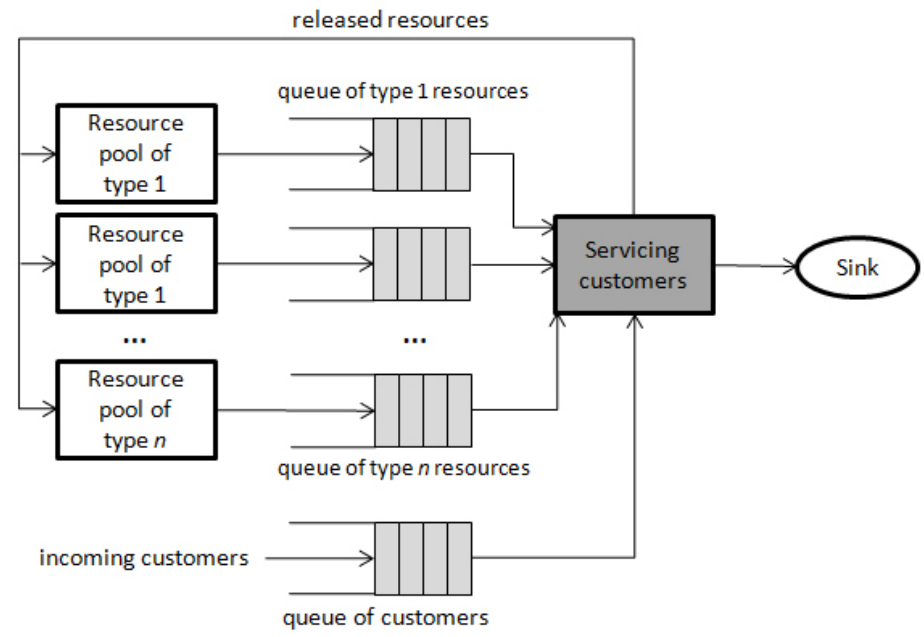

Figure 1. Illustration of the system architecture

\section{Example 1}

In this example our goal was to estimate a deterministic state space model describing the relation between the average waiting time of resources $m_{r}(t)$ and the average waiting 
time of demands $m_{d}(t)$. Before going further let us show the configuration of the analyzed system:

- $N_{R}=3$

- $L_{q}=3$

- $L_{c}=1000$

- $T_{S}$ is an exponential random variable with mean $E\left[T_{S}\right]=3$

- Arrival of demands $d(t)$ : pseudo-random binary sequence (customer generation event is triggered at rising and falling edges)

- Limit of simultaneously serviced customers: 3

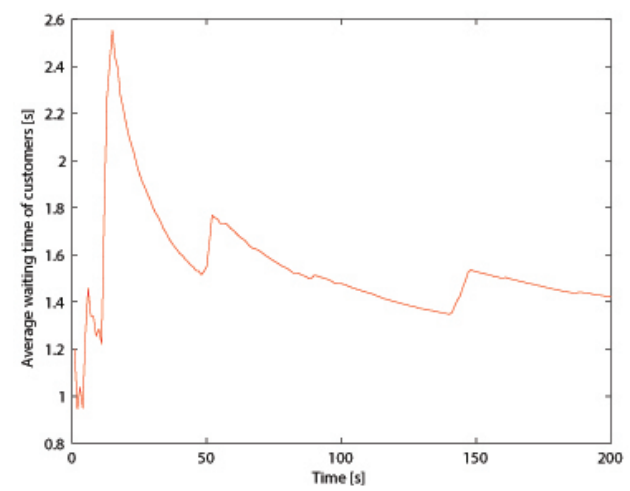

Figure 2. The transformed input $m_{d}(t)$

By considering the above configuration the relation between the two mentioned features of the system has been modeled by subspace identification technique (considering the deterministic case). Fig. 2 shows the input signal reflecting the average waiting time of demands in the corresponding queue. Furthermore, in Fig. 3 the measured and modeled average waiting time of resources in the resource queue can be followed. It is clear that the input in this case stands for a transformed input namely $m_{d}(t)$. In the first parts of the experiment 200 input-output pairs have been used (generated by the above system) for model estimation. The system matrices have been determined based on the described subspace identification approach. As it can be seen the obtained model nicely follows the characteristics of the measured output. In the second part of this experiment only the first 100 input-output pairs have been considered during the model estimation and the rest 100 pairs have been used for validation (see Fig. 4). As reflected by Fig. 4 also in this case the model output nicely follows the characteristics of the measured data. On the other hand if the incoming rate of customers $d(t)$ is considered as input (a pseudo-random number 

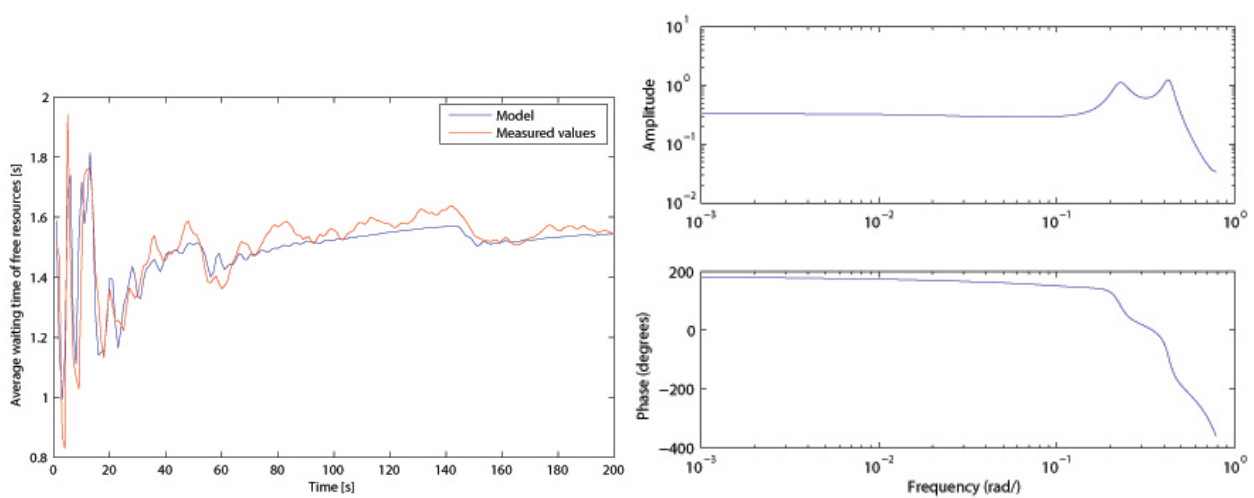

Figure 3. The measured average waiting time of resources $m_{r}(t)$ (red), the output of the identified model (blue), Input: $m_{d}(t)$ (left), Bode diagram of the identified model (right)
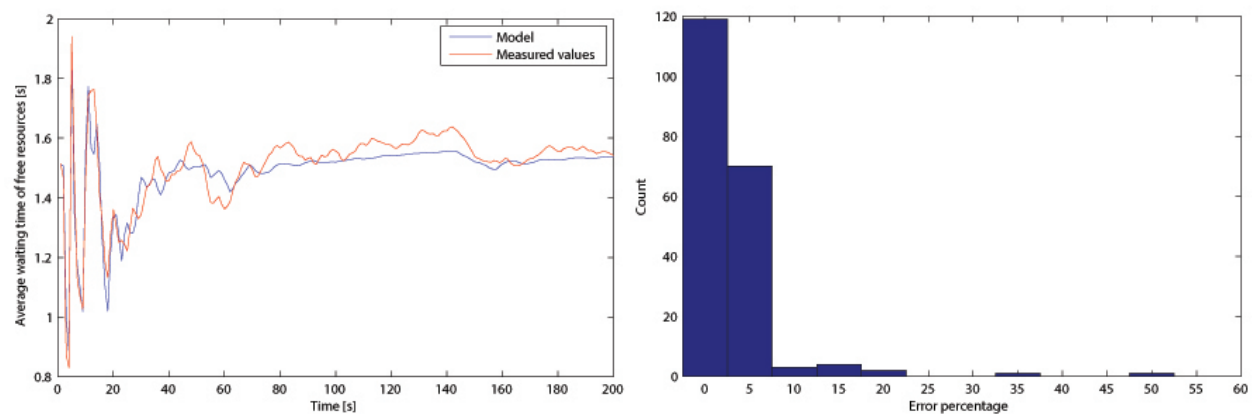

Figure 4. Validation of the identified model. The measured average waiting time of resources $m_{r}(t)(r e d)$, the output of the identified model (blue), Input: $m_{d}(t)$ (the first half of samples has been used for model estimation while the second half for validation (left), Error percentage histogram corresponding to the estimated model (right)

in our case) the obtained model is inaccurate (see Fig. 5). The primary reason for this is the length of queues and the related long delays in the system. If the maximal length of the customer queue is set to a smaller value, more accurate model is obtained. It can be assumed that the accuracy of the obtained model is strongly influenced by the strength of dependence between the two selected features. 

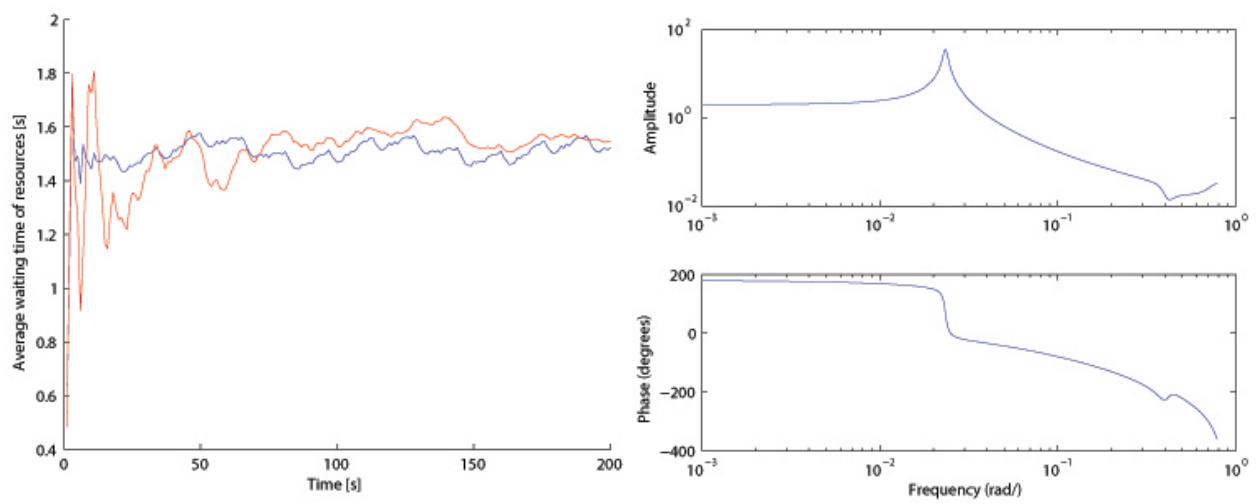

Figure 5. The measured average waiting time of resources $m_{r}(t)$ (red), the output of the identified model (blue), Input: $d(t)$. As it can be seen, the model is of low accuracy which indicates weak relation between the used input-output variables (left), Bode diagram corresponding to the model (right)

\section{Example 2}

The main goal was to model the number of entities in the queue of resources. The input in this example is $T_{S}$ while the output the number of available resources waiting in the queue. Let us consider the following system configuration:

- $N_{R}=40$

- $L_{q}=40$

- $L_{c}=1000$

- $T_{S}$ is an exponential random variable with mean $E\left[T_{S}\right]=3$

- Arrival of customers: pseudo-random binary sequence (customer generation event is triggered at rising and falling edges)

- Limit of simultaneously serviced customers: 40

The input signal can be followed in Fig. 6. It represents the duration of the servicing assigned to a given demand over time. In the first part of this experiment - similarly to the previous example - 200 input-output pairs have been used for model estimation. The measured number of free resources over time together with the output of the identified model can be followed in Fig. 7. The matrices of the obtained deterministic state space 


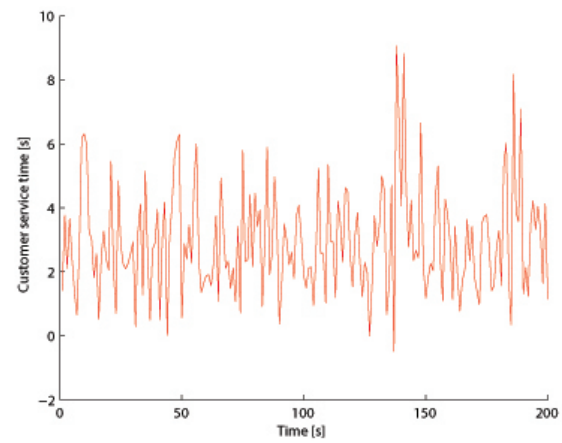

Figure 6. The input signal $T_{S}$

model are as follows:

$$
\begin{aligned}
& \mathbf{A}=\left[\begin{array}{ccc}
0.41814 & -0.080494 & -0.28302 \\
-0.27051 & -0.86992 & -0.066373 \\
0.16624 & -0.36498 & -0.27266
\end{array}\right], \mathbf{B}=\left[\begin{array}{c}
0.033479 \\
0.014832 \\
0.0090137
\end{array}\right], \\
& \mathbf{C}=\left[\begin{array}{lll}
-8.94 & 0.51886 & -1.5867
\end{array}\right], \mathbf{D}=[0], \mathbf{X}=\left[\begin{array}{c}
0.77666 \\
-0.31588 \\
1.1808
\end{array}\right]
\end{aligned}
$$
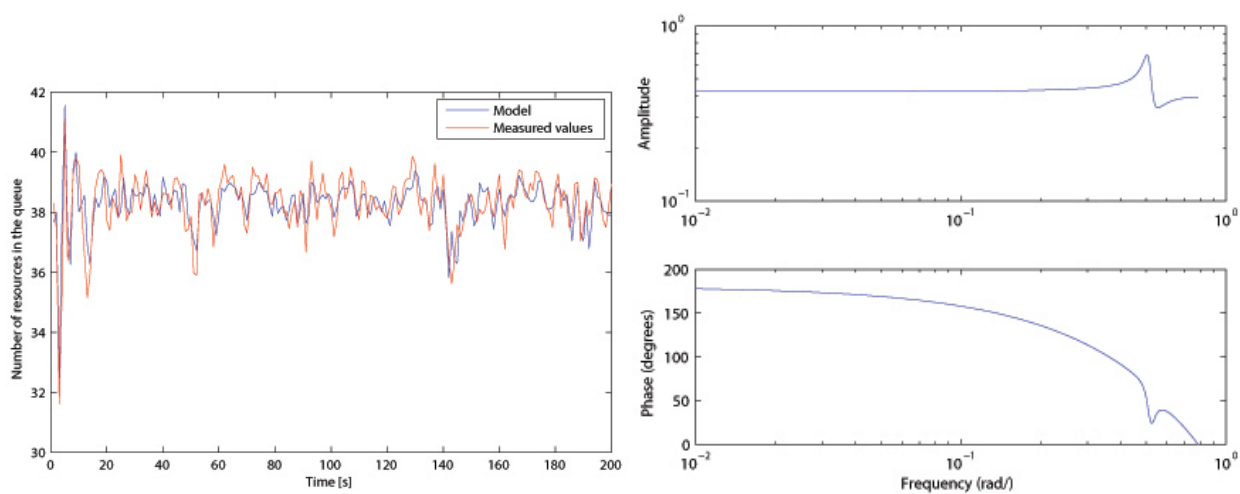

Figure 7. The measured number of free resources (red), output of the identified model (blue), Bode diagram of the identified model (right)

In the second part of the experiment - also similarly to the previous example - only the first 100 input-output pairs have been considered during the model estimation and 

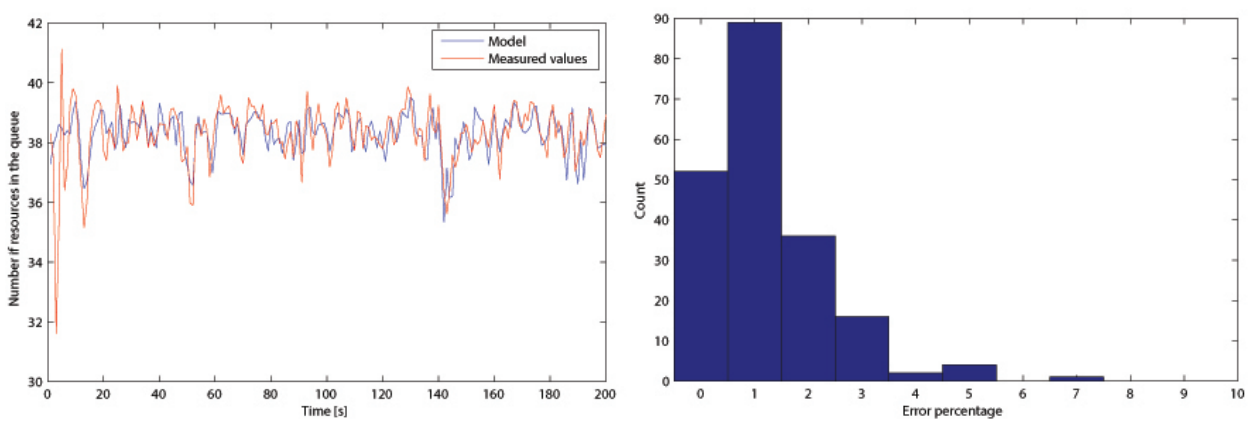

Figure 8. Validation of the identified model. The measured number of free resources (red), output of the identified model (blue) (left), Error percentage histogram corresponding to the estimated model (right)

the rest 100 pairs have been used for validation. The obtained model nicely follows the characteristics of the measured output in both cases (see Fig. 8). The error percentage histogram in Fig. 8 well reflects the accuracy of the estimated model.

\section{Queuing Systems in LPV Framework}

In this section let us give a brief description how queuing systems can be described by linear parameter varying models. It is clear that in complex queuing systems there might be many parameters which may strongly affect the behavior of the whole system. If we discretize the parameter space over a hyper-rectangular grid, for each grid point a linear time invariant (LTI) model can be assigned. The global behavior of the system can be obtained by "blending" the local models properly. Depending on the number of discretization points the number of identified local models might be significant, thus to reduce their number by keeping the accuracy at acceptable level plays another important task in this field [5]. Here the higher order singular value decomposition (HOSVD) plays significant role [4].

Let us consider the following linear parameter varying (LPV) state-space model [5]:

$$
\left(\begin{array}{l}
\dot{\mathbf{x}}(t) \\
\mathbf{y}(t)
\end{array}\right)=\mathbf{S}(\mathbf{p}(t))\left(\begin{array}{l}
\mathbf{x}(t) \\
\mathbf{u}(t)
\end{array}\right),
$$

where $\mathbf{u}(t) \in \mathbb{R}^{u}$ stands for the system input, $\mathbf{y}(t) \in \mathbb{R}^{v}$ represents the output of the system and $\mathbf{x}(t) \in \mathbb{R}^{k}$ denotes the state vector. Furthermore $\mathbf{p}(t)=\left(p_{1}(t), \cdots, p_{N}(t)\right) \in$ $\Omega$,

$$
\Omega=\left[a_{1}, b_{1}\right] \times\left[a_{2}, b_{2}\right] \times \cdots \times\left[a_{N}, b_{N}\right] \subset \mathbb{R}^{N},
$$


and

$$
\mathbf{S}(\mathbf{p}(t))=\left(\begin{array}{ll}
\mathbf{A}(\mathbf{p}(t)) & \mathbf{B}(\mathbf{p}(t)) \\
\mathbf{C}(\mathbf{p}(t)) & \mathbf{D}(\mathbf{p}(t))
\end{array}\right) \in \mathbb{R}^{(k+v) \times(k+u)} .
$$

$\mathbf{S}(\mathbf{p}(t))$ for arbitrary $\mathbf{p}(t)$ parameter vector can be expressed in tensor product form as follows:

$$
\left(\begin{array}{c}
\dot{\mathbf{x}}(t) \\
\mathbf{y}(t)
\end{array}\right)=\left(\mathbf{S} \otimes_{n=1}^{N} \mathbf{w}_{n}^{T}\left(p_{n}\right)\right)\left(\begin{array}{c}
\mathbf{x}(t) \\
\mathbf{u}(t)
\end{array}\right),
$$

where vector $\mathbf{w}_{n}^{T}\left(p_{n}\right) \in \mathbb{R}^{I_{n}}, n=1 . . N$, contains continuous and bounded functions $w_{n, i_{n}}\left(p_{n}\right)$ on interval $\left[a_{n}, b_{n}\right], i_{n}=1 . . I_{n}$. The $(N+2)$-dimensional tensor $\mathbf{S} \in \mathbb{R}^{I_{1} \times \cdots \times I_{N+2}}$ contains the system matrices of linear time invariant vertex systems [5]:

$$
\begin{gathered}
\mathbf{S}_{i_{1} \cdots i_{N}}=\left\{S_{i_{1} \cdots i_{N}, \alpha, \beta}, 1 \leq \alpha \leq I_{N+1}, 1 \leq \beta \leq I_{N+2}\right\} \\
\mathbf{S}_{i_{1} \cdots i_{N}} \in \mathbb{R}^{I_{N+1} \times I_{N+2}}
\end{gathered}
$$

Applying HOSVD on the first $N$ dimensions of $S$ we obtain the following:

$$
\begin{aligned}
& \left(\begin{array}{c}
\dot{\mathbf{x}}(t) \\
\mathbf{y}(t)
\end{array}\right)=\left[\left(\begin{array}{ll}
\mathbf{D} \bigotimes_{n=1}^{N} & \left.\mathbf{U}_{n}\right) \bigotimes_{n=1}^{N} \mathbf{w}_{n}^{T}\left(p_{n}\right)
\end{array}\right]\left(\begin{array}{c}
\mathbf{x}(t) \\
\mathbf{u}(t)
\end{array}\right)\right. \\
& \left(\begin{array}{c}
\dot{\mathbf{x}}(t) \\
\mathbf{y}(t)
\end{array}\right)=[\mathbf{D} \otimes_{n=1}^{N} \underbrace{\mathbf{U}_{n} \mathbf{w}_{n}^{T}\left(p_{n}\right)}_{\tilde{\mathbf{w}}_{n}^{T}\left(p_{n}\right)}]\left(\begin{array}{c}
\mathbf{x}(t) \\
\mathbf{u}(t)
\end{array}\right),
\end{aligned}
$$

where $\mathbf{D}$ stands for the core tensor, and functions $\tilde{w}_{n, j_{n}}\left(p_{n}\right)$ are the weighting functions [5]In order to reduce the number of LTI vertex systems the rightmost columns of matrices $\mathbf{U}_{n}$ may be removed. Some recent applications related to LPV systems and TP model transformation can be found in [11],[12].

\section{Future work and Conclusions}

In the present paper a queuing approach for modeling logistical processes has been proposed. It was shown how the relation between parameters of such queuing models can be identified on subspace basis. The results clearly reflect that in case of strong relation between two system features the identified model nicely approximates the modeled system. On the other hand if this relation is weak the identified model (based on the corresponding input-output pairs) reflects significantly lower accuracy. In addition it was briefly shown how complex logistical systems could be modeled on LPV basis by blending locally identified linear models together. In our case the vertex systems stand for state space models identified based on input-output data. These models are then embedded into tensor representation and transformed into tensor product form. In such form model reduction can also be directly executed. 


\section{References}

[1] van Overschee P, de Moor BL: Subspace Identification for Linear Systems: Theory Implementation - Applications. Kluwer Academic Publishers, 2011.

DOI: $10.1007 / 978-1-4613-0465-4$

[2] Harmati I, Orbán G, Várlaki P: Takagi-Sugeno Fuzzy Control Models for Large Scale Logistics Systems. International Symposium on Computational Intelligence and Intelligent Informatics, Agadir, pp. 199-203, 2007.

DOI: 10.1109/ISCIII.2007.367389

[3] Orbán G, Várlaki P: Fuzzy Modelling for Service Strategy and Operational Control of Loading Systems. Acta Technica Jaurinensis, Series Logistics, Vol. 2, No. 3, pp. 375-391, 2009.

[4] De Lathauwer L, De Moor B, Vandewalle J: A multilinear singular value decomposition. SIAM Journal on Matrix Analysis and Applications, Vol. 21, No. 4, pp. 1253-1278, 2000.

DOI: $10.1137 / \mathrm{S} 0895479896305696$

[5] Szeidl L, Várlaki P: HOSVD Based Canonical Form for Polytopic Models of Dynamic Systems. Journal of Advanced Computational Intelligence and Intelligent Informatics, Vol. 13, No. 1, pp. 52-60. 2009.

[6] Nagy S, Petres Z, Baranyi P: TP Tool - a MATLAB Toolbox for TP Model Transformation. Proceedings of 8th International Symposium of Hungarian Researchers on Computational Intelligence and Informatics, budapest, pp. 483-495, 2007.

[7] Szeidl L, Baranyi P, Petres Z, Várlaki P: Numerical Reconstruction of the HOSVD Based Canonical Form of Polytopic Dynamic Models. International Symposium on Computational Intelligence and Intelligent Informatics, Agadir, pp. 111-116, 2007. DOI: 10.1109/ISCIII.2007.367372

[8] Gunasekaran A, Patel C, McGaughey RE: A framework for Supply Chain Performance Measurement. International Journal of Production Economics, Vol. 87, No 3, pp. 333-347, 2004.

DOI: $10.1016 /$ j.ijpe.2003.08.003

[9] Yao JS, Lin FT: Constructing a Fuzzy Flow-Shop Sequencing Model Based on Statistical Data. International Journal of Approximate Reasoning, Vol. 29, No 3, pp. 215-234, 2002.

DOI: $10.1016 / \mathrm{S} 0888-613 \mathrm{X}(01) 00064-0$

[10] Sevastjanov PV, Róg P: Fuzzy Modeling of Manufacturing and Logistic Systems. Mathematics and Computers in Simulation, Vol. 63, No. 6, pp. 569-585, 2003. DOI: $10.1016 / \mathrm{S} 0378-4754(03) 00064-8$ 
[11] Baranyi P, Petres Z, Korondi P, Yam Y, Hashimoto H: Complexity Relaxation of the Tensor Product Model Transformation for Higher Dimensional Problems. Asian Journal of Control, Vol. 9, No. 2, pp. 195-200, 2007.

DOI: 10.1111/j.1934-6093.2007.tb00323.x

[12] Baranyi P, Tikk D, Yam Y, Patton RJ: From Differential Equations to PDC Controller Design via Numerical Transformation. Computers in Industry, Vol. 51, No. 3, pp. 281-297, 2003.

DOI: $10.1016 / \mathrm{S} 0166-3615(03) 00058-7$ 